\title{
Rescue of Impaired Long-Term Facilitation at Sensorimotor Synapses of Aplysia following siRNA Knockdown of CREB1
}

\author{
Lian Zhou, Yili Zhang, Rong-Yu Liu, Paul Smolen, ㄴoonard J. Cleary, and John H. Byrne \\ Department of Neurobiology and Anatomy, The University of Texas Medical School at Houston, Houston, Texas 77030
}

\begin{abstract}
Memory impairment is often associated with disrupted regulation of gene induction. For example, deficits in cAMP response elementbinding protein (CREB) binding protein (CBP; an essential cofactor for activation of transcription by CREB) impair long-term synaptic plasticity and memory. Previously, we showed that small interfering RNA (siRNA)-induced knockdown of CBP in individual sensory neurons significantly reduced levels of CBP and impaired 5-HT-induced long-term facilitation (LTF) in sensorimotor cocultures from Aplysia. Moreover, computational simulations of the biochemical cascades underlying LTF successfully predicted training protocols that restored LTF following CBP knockdown. We examined whether simulations could also predict a training protocol that restores LTF impaired by siRNA-induced knockdown of the transcription factor CREB1. Simulations based on a previously described model predicted rescue protocols that were specific to CREB1 knockdown. Empirical studies demonstrated that one of these rescue protocols partially restored impaired LTF. In addition, the effectiveness of the rescue protocol was enhanced by pretreatment with rolipram, a selective cAMP phosphodiesterase inhibitor. These results provide further evidence that computational methods can help rescue disruptions in signaling cascades underlying memory formation. Moreover, the study demonstrates that the effectiveness of computationally designed training protocols can be enhanced with complementary pharmacological approaches.
\end{abstract}

\section{Introduction}

Specific defects in biochemical pathways are correlated with impairments in human cognition, learning, and memory (Raymond and Tarpey, 2006; Roelfsema and Peters, 2007; Saura and Valero, 2011; Stornetta and Zhu, 2011; Wijetunge et al., 2013). Animal models of molecular deficits mimic these impairments and are critical to test the efficacy of potential treatments. For example, cAMP response element-binding protein (CREB) binding protein (CBP) deficits impair hippocampal long-term potentiation (LTP) and long-term memory (LTM) (Wood et al., 2005; Barrett et al., 2011), and CBP mutations are the most common cause of Rubinstein-Taybi syndrome (RTS). CBP mutant mice serve as models of RTS (Bourtchouladze et al., 2003; Alarcón et al., 2004; Korzus et al., 2004). In these mice, some memory impairment is rescued by treatment with rolipram, an inhibitor of cAMP phosphodiesterase 4 (PDE4). Rolipram also rescues cognitive deficits in mouse models of Alzheimer's disease and traumatic brain injury (Gong et al., 2004; Titus et al., 2013), and enhances LTP and hippocampal-dependent memory (Barad et al., 1998; Bourtchouladze et al., 2003). The facilitatory effects of rolipram are presumably due to an enhancement of protein ki-

Received Aug. 8, 2014; revised Nov. 20, 2014; accepted Nov. 26, 2014.

Author contributions: L.Z., Y.Z., R.-Y.L., P.S., and J.H.B. designed research; L.Z. and Y.Z. performed research; L.Z., Y.Z., R.-Y.L., P.S., L.J.C., and J.H.B. analyzed data; L.Z., Y.Z., R.-Y.L., P.S., L.J.C., and J.H.B. wrote the paper.

This work was supported by National Institutes of Health grants R01 NS073974 and R01 NS019895. We thank D. Baxter for assistance with preparing the illustrations and E. Kartikaningrum and J. Liu for preparing the cultures.

The authors declare no competing financial interests.

Correspondence should be addressed to Dr. John H. Byrne, Department of Neurobiology and Anatomy, The University of Texas Medical School at Houston, P.0. Box 20708, Houston, TX 77225. E-mail: John.H.Byrne@uth.tmc.edu.

DOI:10.1523/JNEUROSCI.3330-14.2015

Copyright (C) 2015 the authors $\quad 0270-6474 / 15 / 351617-10 \$ 15.00 / 0$ nase A (PKA) activity, thereby increasing phosphorylation of CREB by PKA (MacKenzie and Houslay, 2000; Bourtchouladze et al., 2003).

The use of computational simulations to design optimal training protocols is an alternative, but not necessarily mutually exclusive, approach for the enhancement of memory. For example, a model that describes the dynamics of the cAMP/PKA and rapidly accelerated fibrosarcoma (Raf)/MEK/ERK signaling pathways, both of which are essential for LTF (Martin et al., 1997; Chain et al., 1999), predicted that specific, irregular spacing between trials would maximize the production of key downstream transcription factors and thereby enhance LTF and the formation of LTM (Zhang et al., 2012). These factors include the phosphorylated, activated forms of CREB1 (Bartsch et al., 1998) and CCAAT/enhancer binding protein (C/EBP) (Alberini et al., 1994). C/EBP, when phosphorylated by ERK, activates genes essential for LTF (Alberini et al., 1994; Yamamoto et al., 1999). ERK also phosphorylates the repressor CREB2, relieving repression of transcription (Bartsch et al., 1995).

In addition to enhancing normal memory, this computational strategy predicted a rescue protocol that restored a deficit in LTF associated with an in vitro model of RTS. Liu et al. (2013) used small interfering RNA (siRNA) to reduce the basal level of CBP and impair LTF at sensory neuron-motor neuron (SN-MN) synapses of Aplysia. The mathematical model of Zhang et al. (2012) was extended to include the cAMP/PKA and Raf/MEK/ERK pathways, as well as phosphorylation of CREB1, CREB2, and C/EBP. Simulations predicted training protocols that would compensate for the CBP deficit. These rescue protocols induced substantial temporal overlap of CREB1 activation and CREB2 inactivation by repeated, irregularly spaced stimuli. Subsequent 
empirical studies validated the strategy by confirming that one of these rescue protocols restored LTF.

In the present study, we examine whether this strategy can also be used to rescue a deficit in LTF produced by a different molecular lesion, knockdown of CREB1 induced by siRNA. In addition, we examine the effects of combining the traditional pharmacological approach with computationally designed rescue protocols.

\section{Materials and Methods}

Cell culture, siRNA injection, and electrophysiology. SN-MN cocultures from Aplysia were prepared as described previously (Zhang et al., 2012). A single SN and a single L7 MN were plated onto each dish to make $\mathrm{SN}-\mathrm{MN}$ cocultures. Two to three days after plating, the $\mathrm{SN}$ was injected with siRNA ( $5 \mu \mathrm{M})$ following established procedures (Liu et al., 2011a). Pools of CREB1-siRNA (Dharmacon Research) contained four different sequences of siRNA (Liu et al., 2011a). Scrambled siRNA (Dharmacon Research) was used as an injection control (Con-siRNA). After injection, $\mathrm{SN}-\mathrm{MN}$ cocultures were allowed to grow for another $72 \mathrm{~h}$ at $18^{\circ} \mathrm{C}$. Before pretesting the synaptic connection, the growth medium was replaced by a recording solution that consisted of $50 \%$ isotonic $\mathrm{L} 15$ and $50 \%$ artificial seawater [ASW; containing (in $\mathrm{mm}$ ) $450 \mathrm{NaCl}, 10 \mathrm{KCl}, 11 \mathrm{CaCl}_{2}, 29$ $\mathrm{MgCl}_{2}$, and 10 HEPES, $\mathrm{pH}$ 7.6; L15-ASW]. EPSPs were recorded from MNs with 10-20 M $\Omega$ sharp electrodes filled with $3 \mathrm{M}$ potassium acetate. MNs were hyperpolarized to $-90 \mathrm{mV}$ to prevent action potentials. Sensory neurons were stimulated via a blunt patch electrode filled with L15ASW. Cultures were excluded from further analysis if pretest EPSPs were $<5 \mathrm{mV}$ or $>35 \mathrm{mV}$, which tended to generate action potentials after 5-HT treatment (Zhang et al., 2012). Following pretests, SN-MN cocultures were exposed to five $5 \mathrm{~min}$ pulses of $50 \mu \mathrm{M} 5$-HT to induce LTF. Post-test EPSPs were recorded $24 \mathrm{~h}$ after 5-HT treatment. Experiments were done in a blind manner following published procedures (Liu et al., 2013). Data acquisition was performed using pClamp version 10 (Molecular Devices). All experiments were performed at room temperature $\left(20-25^{\circ} \mathrm{C}\right)$

Data analysis and statistics. LTF was quantified as the percentage increase of the post-test EPSP at $24 \mathrm{~h}$ after 5-HT treatment relative to the pretest EPSP. LTF measurements were excluded if values were outside 3 SDs from the mean. Two experiments were excluded based on this criterion. Parametric tests were used in all experiments because data passed the Shapiro-Wilk normality test. Statistical tests were performed using Sigmaplot 11 (Jandel Scientific). Differences between means were considered significant at $p<0.05$.

Drugs. Stock solutions of 5-HT (5 mm; Sigma-Aldrich) were prepared fresh in L15-ASW on the day of the experiment. Rolipram (SigmaAldrich) was prepared as a $20 \mathrm{~mm}$ stock solution in DMSO and stored at $-20^{\circ} \mathrm{C}$. On the day of an experiment, an aliquot of the rolipram stock solution was diluted in L15-ASW to a final concentration of $0.2 \mu \mathrm{M}$. For groups treated with rolipram $(0.2 \mu \mathrm{M})$, the drug was applied $30 \mathrm{~min}$ before 5 -HT or vehicle (Veh) treatment and continued throughout the experiment.

Computational model. The model of Liu et al. (2013) was extended to include the actions of CREB1-siRNA and rolipram. The model describes activation of PKA and ERK, phosphorylation of CREB1 and CREB2, and synthesis and phosphorylation of C/EBP. The output of the model, predictive of the relative strength of LTF, was taken to be the peak level of phosphorylated C/EBP ( $\mathrm{pC} / \mathrm{EBP}$ ), which is a point of convergence for several second-messenger and transcriptional pathways.

With two exceptions, the equations and parameter values of the model were as described previously [Zhang et al. (2012), their Eqs. 1-12; Liu et al. (2013), their Eqs. 1-7]. To simulate the effects of siRNA knockdown, CREB1 [[CREB1 $]_{\text {total }}$ in Eq. 1 of Liu et al. (2013)] was reduced by $15 \%$, which was equivalent to the average empirical siRNA-mediated knockdown (see Results). The reduction of [CREB1 $]_{\text {total }}$ led to a decreased level of $\mathrm{pC} / \mathrm{EBP}$, in agreement with the empirical attenuation of LTF.

In Aplysia, rolipram inhibits an isoform of PDE4 (ApPDE4) (Park et al., 2005). To simulate the effect of rolipram on LTF, the equation describing the synthesis and degradation of cAMP was modified as follows: $d[c A M P] / d \mathrm{t}=\lambda[5-H T] /\left([5-H T]+K_{5-\mathrm{HT}}\right)-k_{\mathrm{b}, \mathrm{cAMP}}[P D E][c A M P]$. In the equation, $P D E$ represents ApPDE4. The initial concentration of $P D E$ was set as $1 \mu \mathrm{M}$. The effect of rolipram was represented by the reduction of PDE (see Fig. 2A).

The model was programmed in XPPAUT 5.41 (www.math.pitt. edu/ bard/xpp/xpp.html). Fourth-order Runge-Kutta integration was used, with a time step of $3 \mathrm{~s}$. Source codes are available upon request. The model has been uploaded to the ModelDB database (https://senselab. med.yale.edu/modeldb/default.asp, model number 149162).

\section{Results}

\section{Knockdown of CREB1 expression by siRNA blocked LTF}

Previously, we showed that LTF was blocked by injection of CREB1-siRNA within $12 \mathrm{~h}$ after a standard protocol of 5-HT treatment (Liu et al., 2011a). Here, "standard" protocol refers to five 5 min pulses of $50 \mu \mathrm{M} 5$-HT, regularly spaced with an interstimulus interval (ISI) of $20 \mathrm{~min}$ (Montarolo et al., 1986). The siRNA specifically blocked 5-HT-induced increases in CREB1, but basal levels of CREB1 were not affected at $48 \mathrm{~h}$ after siRNA injection. At $72 \mathrm{~h}$ after injection, however, CREB1-siRNA significantly reduced basal levels of CREB1 by $15 \pm 4 \%$ (mean \pm SEM) compared to the control siRNA injections (Liu et al., 2011a). To test whether this reduction of basal CREB1 at $72 \mathrm{~h}$ after injection would impair LTF, empirical studies were performed in four groups of SN-MN cocultures (Fig. 1). In Groups 1 and 3, SNs were injected with control (scrambled) siRNA and treated with either the standard (ST) protocol of 5-HT (50 $\mu \mathrm{M})$ or with equivalent applications of vehicle (Fig. 1A, ST + Con-siRNA, black traces; Veh + Con-siRNA, blue traces). In Groups 2 and 4, SNs were injected with CREB1-siRNA and treated with either the standard protocol or with vehicle (Fig. 1A, ST + CREB1-siRNA, red traces; Veh + CREB1-siRNA, yellow traces). LTF was evaluated $24 \mathrm{~h}$ after application of 5-HT. In the 5-HT treatment groups, LTF was $184 \pm 18 \%$ of the pretest EPSP with injection of Con-siRNA (ST + Con-siRNA, $n=6$ ), whereas LTF was reduced to $121 \pm 10 \%$ of pretest by injection of CREB1 siRNA (ST + CREB1-siRNA, $n=7$ ). Vehicle treatment with injection of either Con-siRNA or CREB1 siRNA did not produce LTF (Veh + ConsiRNA, $102 \pm 6 \%, n=4$; Veh + CREB1-siRNA, $86 \pm 9 \%, n=5$ ). A one-way ANOVA indicated significant overall differences among treatment groups (Fig. $1 B ; F_{(3,18)}=11.82, p<0.001$ ). Injection of CREB1 siRNA significantly impaired LTF. Post hoc pairwise comparison [Student-Newman-Keuls (SNK) method] indicated that the ST + Con-siRNA group was significantly different from the other three groups (Fig. $1 B$; ST + Con-siRNA vs ST + CREB1-siRNA, $q_{2}=5.44, p<0.001 ;$ ST + Con-siRNA vs Veh + CREB1-siRNA, $q_{4}=7.77, p<0.001$; ST + Con-siRNA vs Veh + Con-siRNA, $\left.q_{3}=6.14, p<0.001\right)$. Moreover, no significant differences were found among the ST + CREB1-siRNA, Veh + Con-siRNA, and Veh + CREB1-siRNA groups (Fig. 1B; ST + CREB1-siRNA vs Veh + CREB1-siRNA, $q_{3}=2.86, p=$ 0.14; ST + CREB1-siRNA vs Veh + Con-siRNA, $q_{2}=1.49, p=$ 0.31; Veh + Con-siRNA vs Veh + CREB1-siRNA, $q_{2}=1.11, p=0.44$ ).

\section{Prediction of a stimulus protocol that rescues LTF}

Induction and phosphorylation of C/EBP represents the effect of convergence of the PKA signaling pathway, which enhances CREB1 phosphorylation, and the ERK signaling pathway, which inactivates CREB2 and phosphorylates C/EBP (Fig. 2A). CREB1 activation, CREB2 inactivation, and C/EBP phosphorylation are all required for LTF (Alberini et al., 1994; Bartsch et al., 1995, 1998; Yamamoto et al., 1999; Guan et al., 2003). Therefore, the increase in $\mathrm{pC} / \mathrm{EBP}$ was considered a correlate of the relative extent of LTF and was used as the output of the mathematical 
A
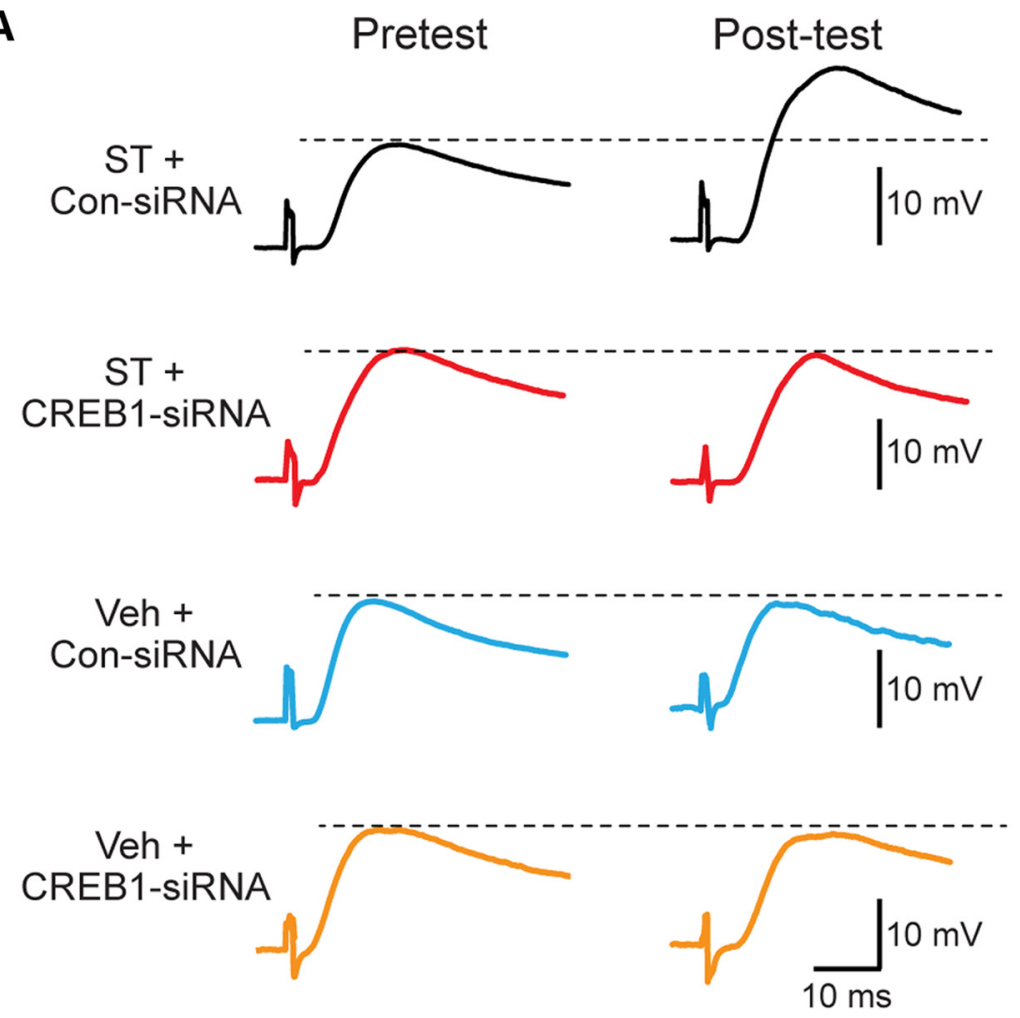

B

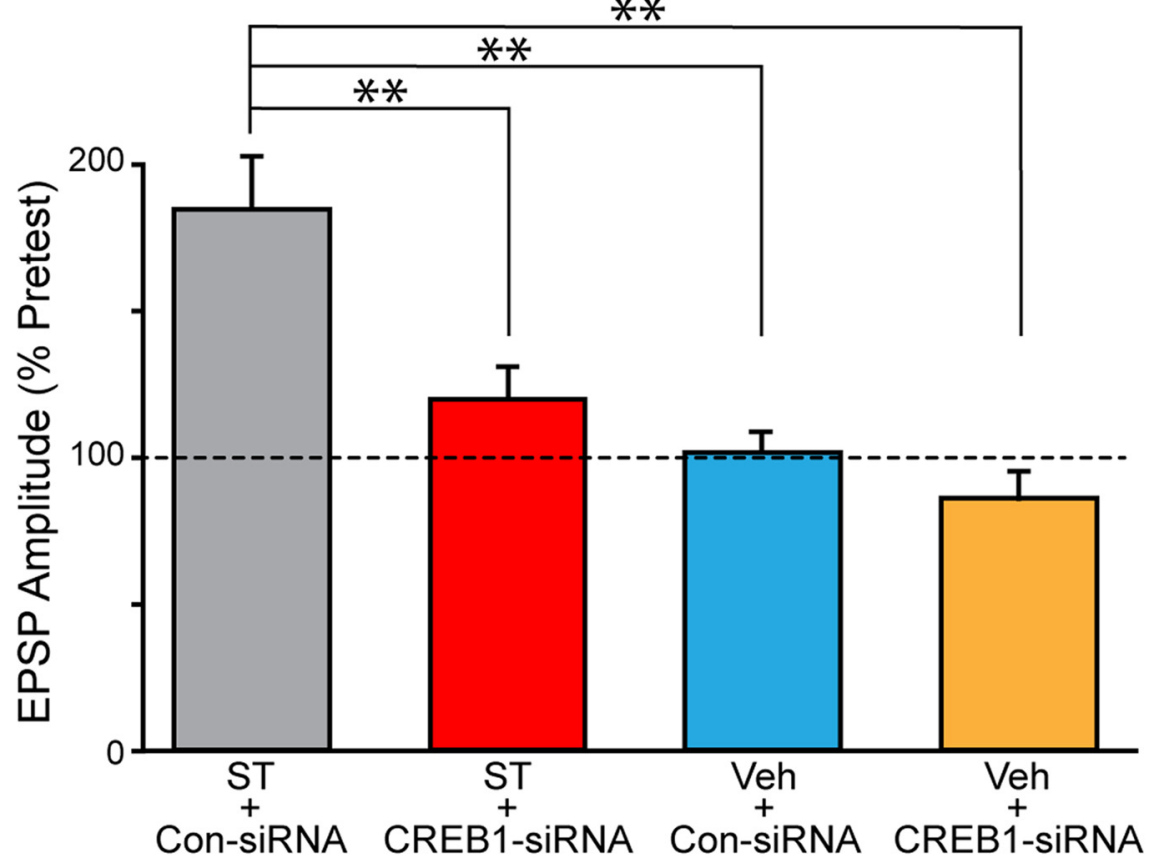

Figure 1. LTF is impaired by siRNA knockdown of CREB1. A, Representative traces of EPSPs immediately before (pretest) and $24 \mathrm{~h}$ after (post-test) the standard 5-HT protocol. SNs were injected with either CREB1 siRNA or Con-siRNA $72 \mathrm{~h}$ before the pretest. Effects of knockdown of CREB1 on LTF were examined in four treatment conditions: a standard protocol of 5-HT treatment in the presence of either (1) Con-siRNA (black trace) or (2) CREB1 siRNA (red trace) and a standard protocol of vehicle treatment in the presence of either (3) Con-siRNA (blue trace) or (4) CREB1 siRNA (yellow trace). In this and subsequent illustrations, the amplitude of pretest EPSPs is indicated by dashed lines. $\boldsymbol{B}$, Summary data for LTF after the standard treatment. In this and subsequent illustrations, LTF was quantified by the percentage increase of the post-test EPSP relative to the pretest EPSP (\% Pretest), and error bars represent the mean \pm SEM. A one-way ANOVA indicated no significant differences in the amplitude of pretest EPSPs among the four groups $\left(F_{(3,18)}=0.209, p=0.89\right)$. For this and subsequent illustrations, asterisks indicate the statistical results from post hoc pairwise comparisons. ${ }^{* *} p<0.01$. model (Liu et al., 2013). To simulate the magnitude of empirical reduction of CREB1 by siRNA, the model parameter [CREB 1$]_{\text {total }}$ was reduced to $85 \%$ of the value used previously (Liu et al., 2013). With this reduction, simulations of the standard 5-HT protocol exhibited a decrease of peak phosphorylated C/EBP (pC/EBP) (Fig. 2B1,B2). The percentage decrease of simulated peak pC/EBP (from $\sim 0.08 \mu \mathrm{M}$ with ST + Con-siRNA to $\sim 0.06$ $\mu \mathrm{M}$ with ST + CREB1-siRNA; $\sim 30 \%$ decrease) was comparable to the empirical impairment of LTF in Figure $1 B$ (from $184 \%$ with ST + Con-siRNA to $121 \%$ with ST + CREB1-siRNA; 34\% decrease). We next attempted to predict a rescue protocol that would compensate for reduced [CREB1] and restore the simulated peak $\mathrm{pC} / \mathrm{EBP}$ to the peak level produced by the standard protocol with normal [CREB1]. After simulation of $10^{4}$ candidate protocols [performed as described by Liu et al. (2013)], 336 protocols were found to restore the simulated peak level of pC/EBP. Among these 336 potential rescue protocols, one was arbitrarily selected for empirical analysis (Fig. 2B3). This protocol had ISIs of $10,15,20$, and $10 \mathrm{~min}$, and is henceforth termed the "rescue protocol" (Res; Fig. 2B3). In simulations, the rescue protocol restored the peak level of $\mathrm{pC} / \mathrm{EBP}$ above the peak level produced by the standard protocol without CREB1 knockdown (Fig. 2B1,B3; Res + CREB1-siRNA, $\sim 0.11 \mu \mathrm{M}$ vs ST + Con-siRNA, $\sim 0.08 \mu \mathrm{M})$. The peak level of $\mathrm{pC} / \mathrm{EBP}$ simulated by the rescue protocol in the absence of CREB1 siRNA was $0.15 \mu \mathrm{M}, \sim 35 \%$ higher than the level in the presence of CREB1 siRNA. In the presence of CREB1 siRNA, the simulated peak level of pCREB 1 was reduced by $\sim 18 \%$ in both the standard and rescue protocols.

Because degradation of cAMP is an important step that limits the effectiveness of the signaling cascade, we hypothesized that the efficacy of the rescue protocol could be further enhanced by treatment with rolipram. As a first step, we simulated the effects of decreased PDE activity (ranging from 0 to $50 \%$ ) on the peak level of pC/EBP in the CREB1 knockdown model with the standard protocol (Fig. 2C, blue). Decreases in PDE activity by more than $\sim 30 \%$ restored the simulated peak $\mathrm{pC} / \mathrm{EBP}$ to or above the level observed without CREB1 knockdown (Fig. 2C, arrow 2). We next simulated the effects of decreases in PDE paired with the rescue protocol (Fig. 2C, red). Decreased PDE further enhanced the peak level of $\mathrm{pC} / \mathrm{EBP}$ induced by the rescue protocol. In addition, modest synergistic effects were observed when rolipram was included with 
the rescue protocol. For example, a $30 \%$ decrease of PDE alone increased the simulated peak level of pC/EBP by $\sim 0.02 \mu \mathrm{M}$ [Fig. 2C; from $0.06 \mu \mathrm{M}$ (arrow 1) to $\sim 0.08$ $\mu \mathrm{M}$ (arrow 2)], whereas the rescue protocol alone increased the peak level of $\mathrm{pC} / \mathrm{EBP}$ by $\sim 0.05 \mu \mathrm{M}$ [from $0.06 \mu \mathrm{M}$ (arrow 1 ) to $\sim 0.11 \mu \mathrm{M}$ (arrow 3 )]. In contrast, combining the $30 \%$ decrease of PDE with the rescue protocol increased the simulated peak level of $\mathrm{pC} / \mathrm{EBP}$ by $\sim 0.08$ $\mu \mathrm{M}$ [Fig. $2 C$; from $\sim 0.06 \mu \mathrm{M}$ (arrow 1) to $\sim 0.14 \mu \mathrm{M}$ (arrow 4)]. This combined effect is slightly greater than the $0.07 \mu \mathrm{M}$ increase predicted by simply adding the effects of the PDE decrease alone and the rescue protocol alone.

Figure 3 illustrates the simulated dynamics of active PKA (PKAc), active ERK (pERK), phosphorylated CREB1 (pCREB1) and CREB2 (pCREB2), and pC/EBP. The dashed black traces in Figure 3, $C$ and $E$, represent $\mathrm{pCREB} 1$ and $\mathrm{pC} / \mathrm{EBP}$ in response to the standard protocol after knockdown of CREB1. Solid black traces represent the response of the model to the standard protocol with control levels of CREB1, whereas red traces represent the response to the rescue protocol after knockdown of CREB1. Compared to immediate activation of PKA by 5-HT, 5-HT elicits a delayed, transient activation of ERK (Philips et al., 2013). The early, shorter ISIs in the rescue protocol, between pulses 1 and 2 and pulses 2 and 3, triggered an earlier phosphorylation and activation of ERK (Fig. 3B), and therefore a more rapid phosphorylation of CREB2, relieving its repression of $c / e b p$ expression (Fig. 3D). Concurrently, all the 5-HT pulses in the rescue protocol rapidly activated $\mathrm{PKA}$, which rapidly phosphorylated CREB1 (Fig. 3A,C). Pulses 4 and 5 were separated from pulses $1-3$ by a longer ISI (Fig. 3F). As a result, the increase in pCREB1 induced by pulses 4 and 5 overlaps more effectively with the increase in pCREB2 induced by pulses 1-3, compared to the standard protocol (Fig. $3 C, D)$. Pulses 4 and 5 also acted to prolong ERK activation, so that substantial phosphorylation of newly synthesized C/EBP could occur (Fig. $3 B, E$ ).

\section{Rescue protocol partially restored impaired LTF}

To validate the effectiveness of the rescue protocol (ISIs, 10, 15, 20, and $10 \mathrm{~min}$ ), empirical studies were performed in three groups of SN-MN cocultures (Fig. 4). In Group 1, SNs were injected with control siRNA and treated with the standard 5-HT protocol to assess normal LTF (Fig. 4A, ST + Con-siRNA, black traces). In Groups 2 and 3, SNs were injected with CREB1 siRNA
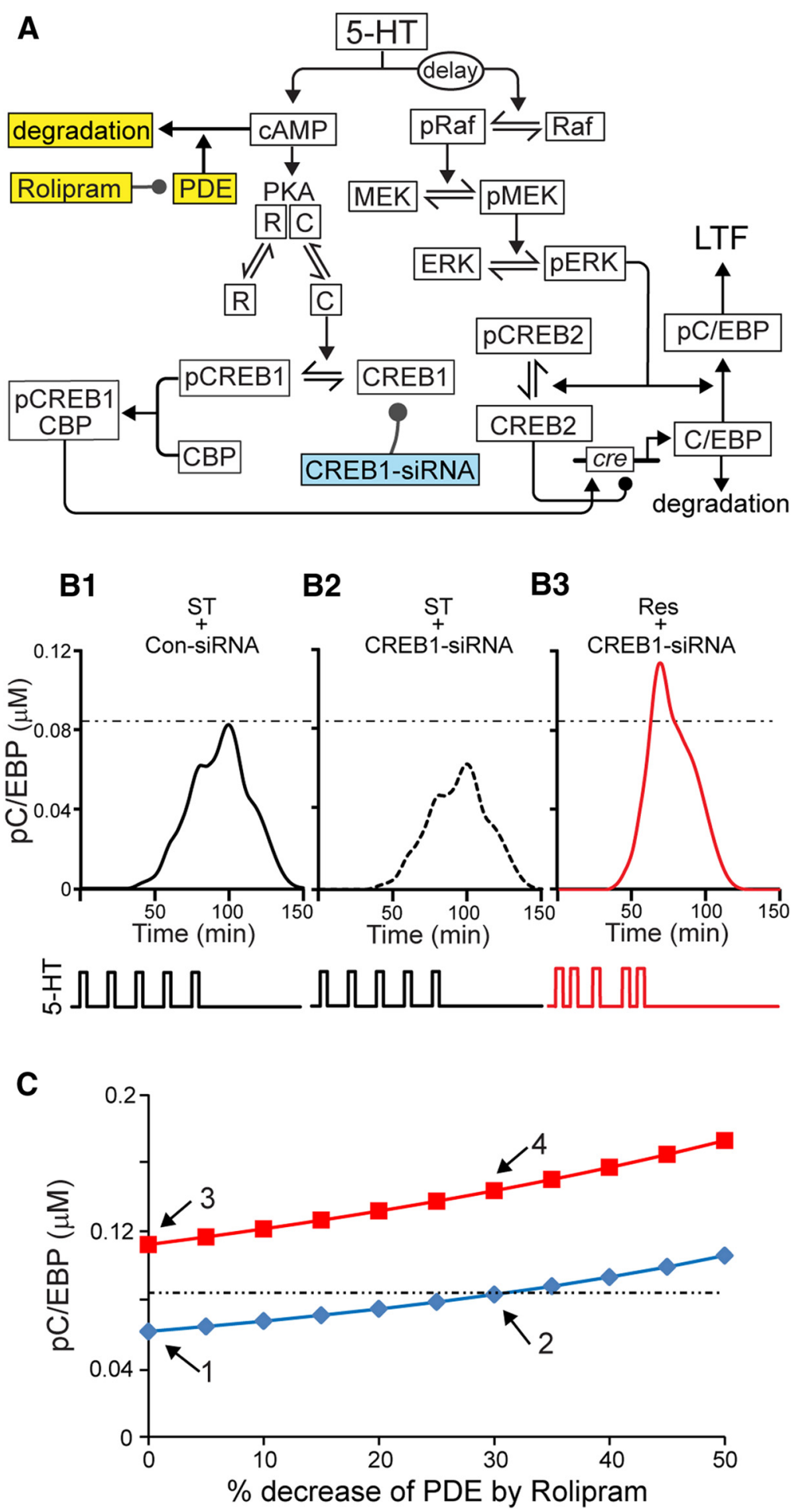

Figure 2. Model of a molecular network underlying LTF. A, The model is modified from Figure $2 A$ in Liu et al. (2013), including the effects of rolipram (yellow components) and CREB1 siRNA (blue components). Arrows and circles indicate positive and negative regulation of transcription and other biochemical processes, respectively. B1, B2, Simulations of the standard protocol with normal CREB1 levels (B1) and with CREB1 knockdown (B2). B3, The rescue protocol (Res) with the CREB1 knockdown boosted pC/EBP levels (red trace) beyond control levels (dashed/dotted line). $\boldsymbol{C}$, Simulations of the effect of rolipram combined with the standard protocol (blue curve) and rescue protocol (red curve) in the presence of CREB1 knockdown. The dashed/dotted line represents the peakpC/EBP level of the standard protocol in the absence of CREB1 siRNA. Arrows 1-4 denote specific PC/EBP levels discussed in the text.

and treated with either the standard protocol (Fig. 4A, ST + CREB1-siRNA, red traces) or the rescue protocol (Res + CREB1siRNA, blue traces). In the standard 5-HT treatment groups, LTF was $156 \pm 9 \%$ of pretest with injection of Con-siRNA (ST + 


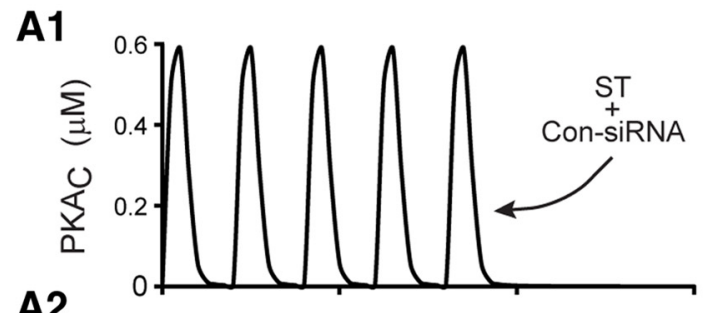

A2

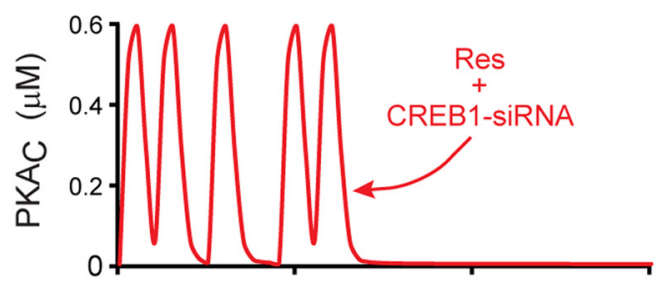

B

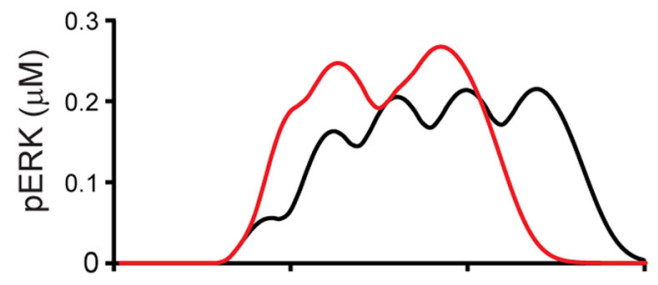

C

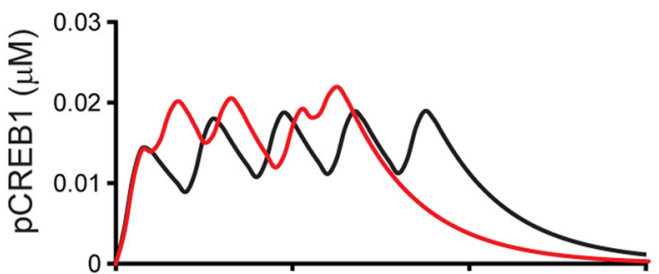

D

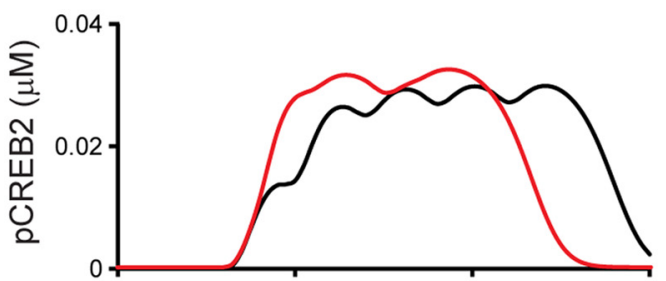

E

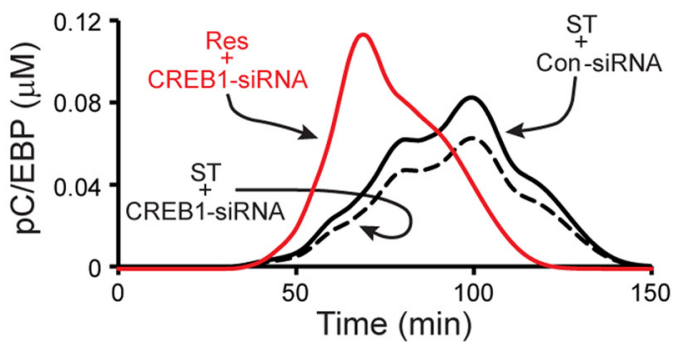

$\mathbf{F}$

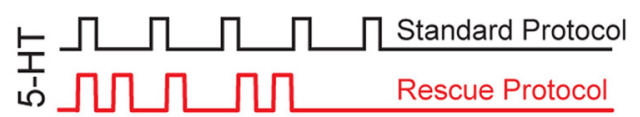

Figure 3. Dynamics of key signaling molecules during induction of LTF. A1-E, Black traces illustrate the responses of the model to the standard protocol, whereas red traces illustrate the responses to the rescue protocol. $\boldsymbol{A 1}, \boldsymbol{A 2}$, Time courses of PKA activity (the concentration of free catalytic subunit, PKA $)$. $\boldsymbol{B}$, Time courses of ERK activity (pERK). $\boldsymbol{C}$, Time courses of active (REB1 (pCREB1). $\boldsymbol{D}$, Time courses of inactive CREB2 (pCREB2). $\boldsymbol{E}$, Time courses of active C/EBP (pC/EBP). The black solid and black dashed time courses in $\boldsymbol{C}$ and $\boldsymbol{E}$ are for the standard protocol without and with CREB1 knockdown, respectively. The solid red traces in $\boldsymbol{C}$ and $\boldsymbol{E}$ are the time courses of the rescue protocol with CREB1 knockdown. $\boldsymbol{F}$, 5-HT pulses and ISIs for the standard protocol (black) and rescue protocol (red). The standard protocol consisted of uniform ISIs of $20 \mathrm{~min}$, whereas the rescue protocol consisted of nonuniform ISIs of 10, 15, 20, and $10 \mathrm{~min}$.
Con-siRNA, $n=12$ ), whereas LTF was reduced to $108 \pm 6 \%$ of pretest by injection of CREB1 siRNA (ST + CREB1-siRNA, $n=$ 9). The rescue protocol partially restored LTF to $133 \pm 7 \%$ of pretest (Res + CREB1-siRNA, $n=11$ ). A one-way ANOVA indicated significant overall differences among treatment groups (Fig. $4 B ; F_{(2,29)}=9.64, p<0.001$ ). Post hoc pairwise comparison (SNK) indicated that LTF in the ST + CREB1-siRNA group was significantly less than in the ST + Con-siRNA group, confirming the effectiveness of the knockdown (Fig. 4B; ST + Con-siRNA vs ST + CREB1-siRNA, $\left.q_{3}=6.2, p<0.001\right)$. In addition, LTF in the Res + CREB1-siRNA group was significantly greater than in the ST + CREB1-siRNA group, but also significantly smaller than in the ST + Con-siRNA group, indicating partial rescue of LTF (Fig. 4B; Res + CREB1-siRNA vs ST + CREB1-siRNA, $q_{2}=3.16, p<0.05$; $\mathrm{ST}+$ Con-siRNA vs Res + CREB1-siRNA, $\left.q_{2}=3.14, p<0.05\right)$.

\section{Effectiveness of the rescue protocol was further enhanced by rolipram}

The model predicted that rolipram would improve the effectiveness of the rescue protocol by increasing cAMP levels. First, we examined the effects of rolipram by itself on LTF. A concentration of $0.2 \mu \mathrm{M}$ was selected because similar concentrations are effective in enhancing LTP (Barad et al., 1998; Titus et al., 2013). Four groups of SN-MN cocultures were examined (Fig. 5). Group 1 was treated with the standard protocol to confirm 5-HTinduced LTF (Fig. 5A, ST, black traces). Group 2 was a control treated with vehicle (Fig. 5A, Veh, red traces). For Groups 3 and 4, rolipram was applied $30 \mathrm{~min}$ before 5 -HT or vehicle and was maintained in the medium until the end of 5-HT/vehicle treatment. LTF was evaluated $24 \mathrm{~h}$ later following application of 5-HT. In the standard 5-HT treatment groups, 5-HT alone yielded LTF of $146 \pm 9 \%$ of pretest (ST, $n=8$ ), and LTF was further enhanced to $165 \pm 8 \%$ of pretest by rolipram (ST + Roli, $n=10)$. In the vehicle and rolipram alone treatment groups, little or no LTF was observed (Veh, $108 \pm 4 \%$ of pretest, $n=9$; Veh + Roli, $120 \pm 4 \%$ of pretest, $n=9)$. A one-way ANOVA indicated significant overall differences among treatment groups (Fig. 5B; $\left.F_{(3,32)}=15.426, p<0.001\right)$. Post hoc pairwise comparison (SNK) indicated that LTF in the ST group was significantly greater than in the Veh and Veh + Roli groups, confirming that 5-HT induced LTF (Fig. $5 B$; ST vs Veh + Roli, $q_{2}=3.7, p<0.05$; ST vs Veh, $\left.q_{3}=5.4, p<0.01\right)$. Rolipram alone did not induce significant LTF as compared to the vehicle group (Veh + Roli vs Veh, $q_{2}=$ $1.79, p=0.22)$. However, LTF in the ST + Roli group was significantly greater than in the other three groups (Fig. $5 B$; $\mathrm{ST}+$ Roli vs ST, $q_{2}=2.9, p<0.05 ;$ ST + Roli vs Veh + Roli, $q_{3}=6.95, p<$ $0.001 ; \mathrm{ST}+$ Roli vs Veh, $\left.q_{4}=8.8, p<0.001\right)$. Therefore, rolipram enhanced 5-HT-induced LTF.

Next, we examined the effects of a combination of the rescue protocol with rolipram on the impaired LTF following CREB1 knockdown. Three groups of SN-MN cocultures were examined (Fig. 6). In all groups, SNs were injected with CREB1 siRNA. Group 1 was treated with the rescue protocol in the presence of $0.2 \mu \mathrm{M}$ rolipram (Fig. $6 A$, Res + Roli + CREB1-siRNA, black traces). Group 2 was treated with the rescue protocol alone (Fig. $6 A$, Res + CREB1-siRNA, red traces). Group 3 was treated with $0.2 \mu \mathrm{M}$ rolipram alone (Fig. $6 \mathrm{~A}$, Veh + Roli + CREB1-siRNA, blue traces). LTF was evaluated $24 \mathrm{~h}$ later following application of 5-HT. Pairing rolipram with the rescue protocol yielded LTF of $158 \pm 9 \%$ of pretest (Res + Roli + CREB1-siRNA, $n=11$ ), whereas the rescue protocol alone produced LTF of $132 \pm 8 \%$ of pretest (Res + CREB1-siRNA, $n=10$ ). Rolipram alone treatment in the presence of injection of CREB1 siRNA did not yield 
significant LTF $(109 \pm 10 \%$ of pretest, Veh + Roli + CREB1-siRNA, $n=9$ ). A one-way ANOVA indicated significant overall differences among treatment groups (Fig. $6 B ; p<0.01$ ). LTF in the Res + Roli group was significantly greater than in the Res alone or Veh + Roli group (Fig. 6B; Res + Roli + CREB1-siRNA vs Veh + Roli + CREB1-siRNA, $q_{3}=5.52$, $p<0.01$; Res + Roli + CREB1-siRNA vs Res + CREB1-siRNA, $\left.q_{2}=3.1, p<0.05\right)$. These results illustrate that the effectiveness of the computational approach can be improved with a complementary pharmacological approach.

\section{Discussion}

The present study examined whether a computationally designed training protocol can be used to rescue a deficit in LTF produced by a knockdown of CREB1 induced by siRNA. In addition, it examined the effects of combining a rescue training protocol with a traditional pharmacological approach.

\section{Rescue of impaired LTF}

Extensive studies have demonstrated that CREB-related signaling plays an essential role in regulating LTM and long-term synaptic plasticity. In Aplysia, injection of creb1 oligonucleotides, or of antibodies to CREB1, blocks 5-HT-induced LTF (Dash et al., 1990; Bartsch et al., 1998), as does injection of CREB1 siRNA (Liu et al., 2011a), a result that was confirmed in the present study (Fig. 1). In rodents, targeted knock-out of CREB isoforms disrupts long-term memory in several behavioral protocols, such as fear conditioning and spatial learning in the water maze (Bourtchuladze et al., 1994; Pittenger et al., 2002; Peters et al., 2009), whereas overexpression of CREB via viral transfection enhances fear conditioning (Josselyn et al., 2001; Restivo et al., 2009). In addition to CREB, CBP is also essential for LTM and long-term synaptic plasticity (Guan et al., 2002; Alarcón et al., 2004; Wood et al., 2005; Barrett et al., 2011). Previously, Liu et al. (2013) showed that the deficit in LTF produced by a reduction of CBP, a cellular model of RTS, could be rescued by a computationally designed training protocol. The present study demonstrates that same strategy can be used to rescue a deficit in LTF produced by a reduction of CREB1 levels.

The simulations of empirically validated rescue protocols for CREB knockdown (Fig. 3) and for CBP knockdown (Liu et al., 2013) suggest that successful establishment of LTM depends on sub-
A
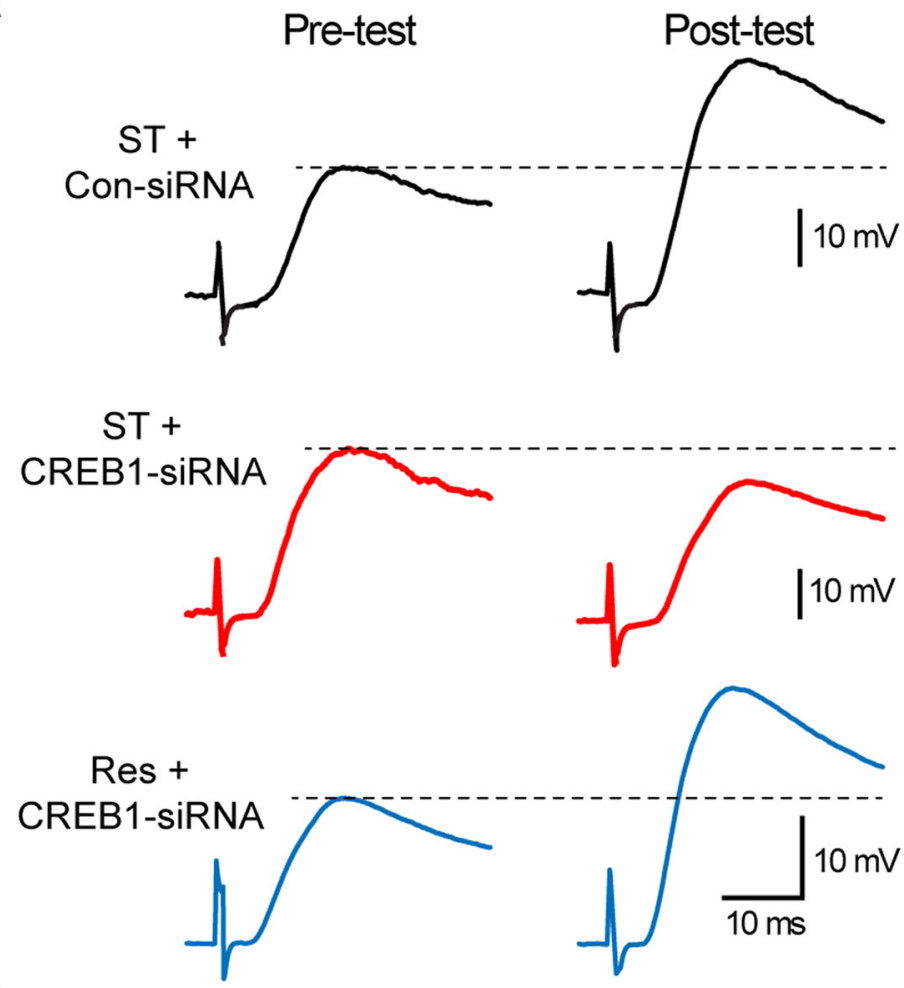

B

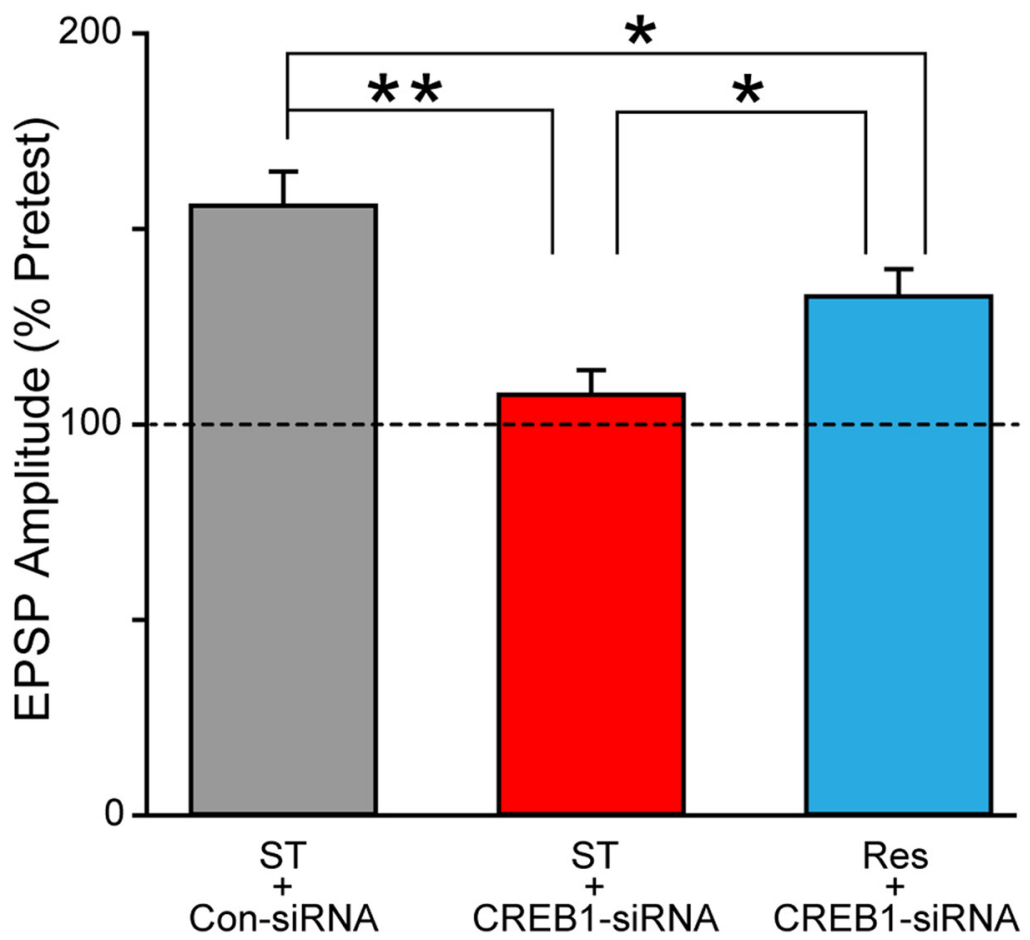

Figure 4. Rescue protocol partially restored impaired LTF. A, Representative traces of EPSPs immediately before (pretest) and $24 \mathrm{~h}$ after (post-test) either the standard or rescue protocols. SNs were injected with either CREB1 siRNA or Con-siRNA $72 \mathrm{~h}$ before the pretest. Three groups were examined: the standard protocol of 5 -HT treatment in the presence of (1) Con-siRNA (black trace) and (2) CREB1 siRNA (red trace), and (3) the rescue protocol of 5-HT treatment in the presence of CREB1 siRNA (blue trace). The initial biphasic responses on the traces are stimulus artifacts. $\boldsymbol{B}$, Summary data. Error bars represent the mean \pm SEM. A one-way ANOVA indicated no significant differences in the amplitude of pretest EPSPs among the three groups $\left(F_{(2,29)}=0.027, p=0.97\right) .{ }^{*} p<0.05 ;{ }^{* *} p<0.01$. 
A
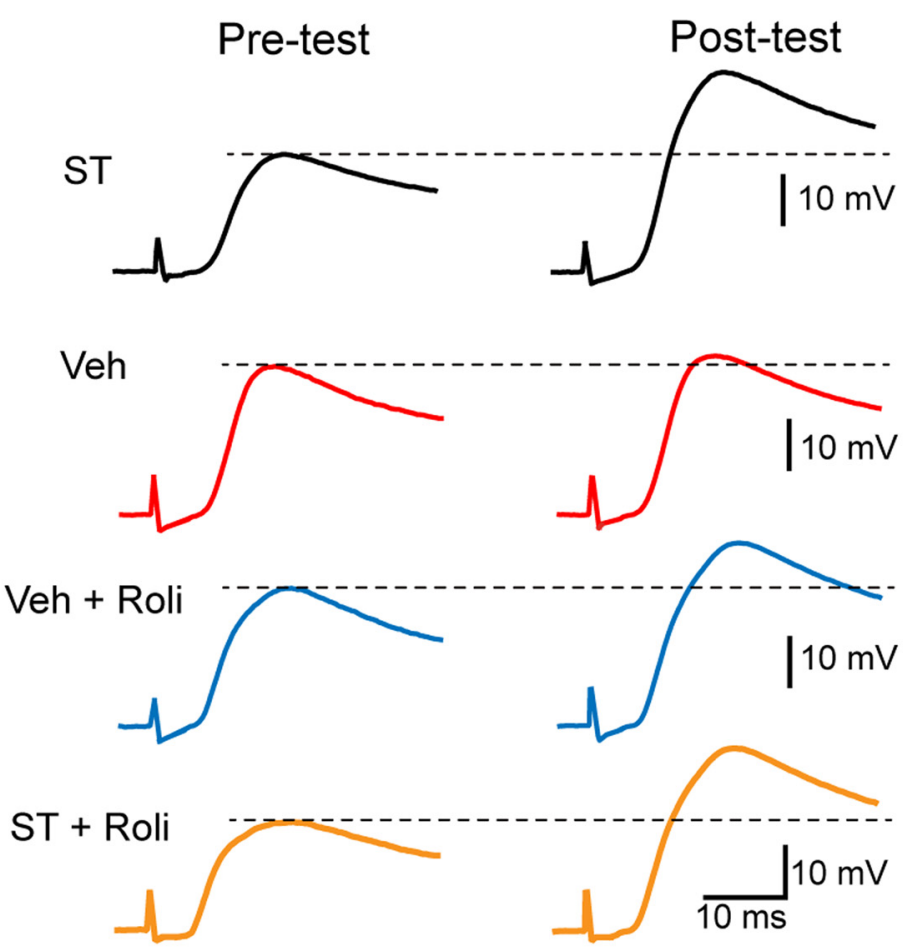

B

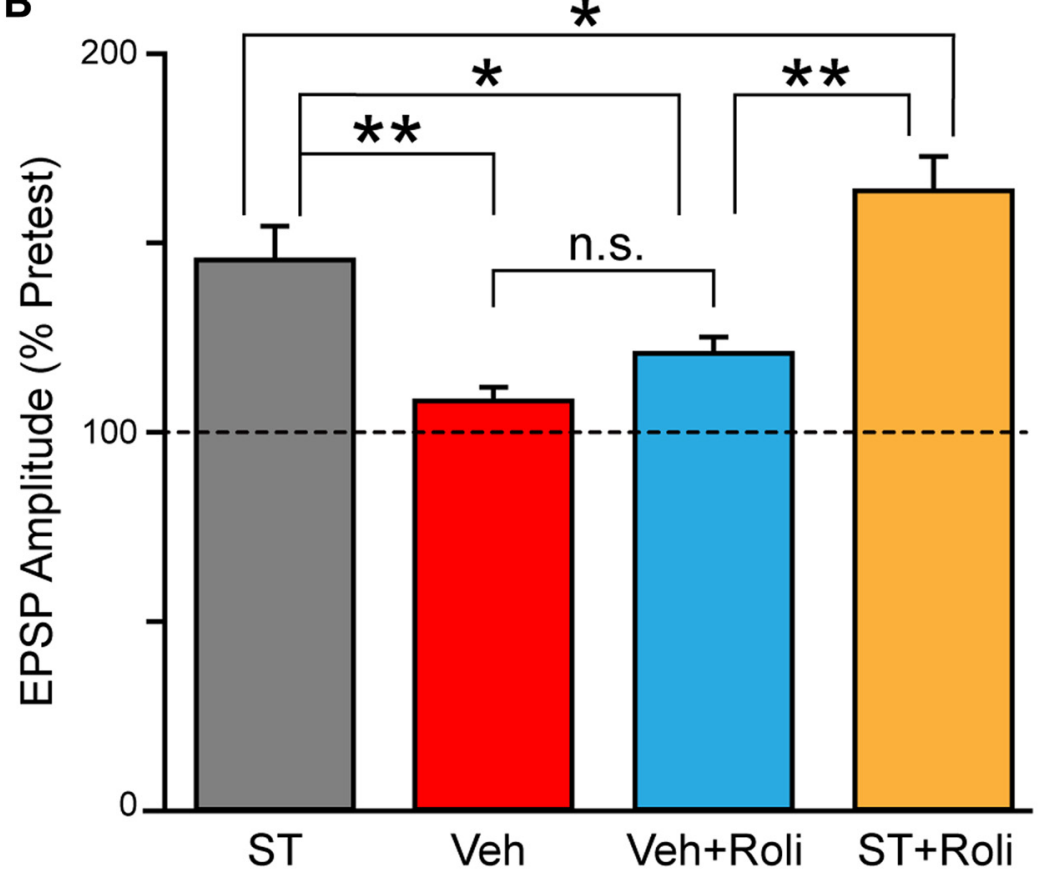

Figure 5. Rolipram enhanced LTF. $A$, Representative traces of EPSPs immediately before (pretest) and $24 \mathrm{~h}$ after (post-test) the standard protocol. In the absence of siRNA injection, effects of rolipram on LTF were examined in four treatment conditions: (1) the standard protocol of 5-HT treatment (black trace), (2) the standard protocol of vehicle treatment (red trace), (3) the standard protocol of vehicle treatment in the presence of rolipram (blue trace), (4) the standard protocol of 5-HT treatment in the presence of rolipram (yellow trace). $\boldsymbol{B}$, Summary data. Error bars represent the mean \pm SEM. A one-way ANOVA indicated that there were no significant differences in the amplitude of pretest EPSPs among the four groups $\left(F_{(3,32)}=0.45, p=0.71\right){ }^{*} p<0.05 ;{ }^{* *} p<0.01$. n.s., Not statistically significant. protocols—specific, irregularly spaced ISI patterns - that optimize such overlap and enhance learning and LTM.

The rescue protocol for CREB1 knockdown, with ISIs of $10,15,20$, and $10 \mathrm{~min}$, is very similar to the previous rescue protocol for CBP knockdown (Liu et al., 2013), which had ISIs of $10,10,20$, and 10 $\mathrm{min}$. In simulations of both rescue protocols, the early short ISIs (relative to the standard protocol ISIs of $20 \mathrm{~min}$ ) yield a more rapid activation of ERK, and therefore a more rapid phosphorylation of CREB2, relieving repression of $c / e b p$ transcription. In both protocols, 5-HT pulses 4 and 5 are separated from pulses $1-3$ by a longer ISI, which acts to create a second, distinct peak of CREB1 phosphorylation, increasing the overlap between CREB1 phosphorylation and CREB2 phosphorylation (Fig. 3). This increase also contributes to $c / e b p$ induction. Indeed, of the 10,000 protocols that were simulated, 336 protocols restored the peak of phosphorylated C/EBP up to or above its normal value. One of these protocols was the CBP rescue protocol in Liu et al. (2013). Therefore, we expect that the CBP rescue protocol would also rescue the LTF deficit induced by CREB1 knockdown. This robustness of rescue protocols presumably occurs because CREB1 and CBP have similar regulatory roles in the CREB signaling pathway, in that CBP is an obligatory cofactor for CREB1. More generally, however, molecular deficits in different molecular pathways would be likely to require distinctively different rescue protocols chosen to interact with the dynamics of the affected pathway in such a manner as to restore, or optimize, pathway output.

\section{Combining a pharmacological approach with a computationally designed rescue protocol}

We also examined whether concurrent enhancement of PKA activity by rolipram could further increase the likelihood of successful rescue of impaired LTF by the rescue protocol alone. We found empirically that pairing rolipram with the rescue protocol significantly enhanced LTF relative to that produced by the rescue protocol alone. Rolipram alone did not produce LTF, perhaps because basal levels of cAMP synthesis did not produce sufficient PKA activation. Combination drug stantial overlap between mutually reinforcing elements of the PKA and ERK signaling pathways. For Aplysia and mammals, these events include PKA-mediated phosphorylation and activation of CREB/CREB1 and C/EBP, reinforced in Aplysia by ERKmediated inactivation of CREB2. The strategy we developed suggests that modeling can successfully predict spaced training therapies that may improve memory and cognition are being investigated for Alzheimer's disease (Aytan et al., 2013; Kawahara et al., 2014) and schizophrenia (Stenberg et al., 2011; Xiao et al. 2012). When developing combined drug therapies, a desirable goal is to obtain synergism, in which the efficacy of paired drugs exceeds the efficacy that would be predicted by summing the 
effect of the individual drugs (Loewe, 1953; Novick, 2013; Zhang et al., 2014). Dose reductions facilitated by synergism may also minimize undesirable off-target effects of the drugs (Zimmermann et al., 2007). An extension of this idea is to combine the use of enhanced training protocols with one or more pharmacological agents. Consequently, we used simulations to predict whether an analogous form of synergism might exist between rolipram and the rescue stimulus protocol. Would the rescue of LTF (i.e., increase in the peak pC/EBP level) due to concurrent application of rolipram and the rescue protocol exceed the sum of the increases produced by these treatments individually? Only a small degree of synergism was predicted by these simulations. Nonetheless, it is likely that replacing the standard protocol with a rescue protocol would reduce the dosage of rolipram required for complete rescue of LTF, thereby reducing off-target drug effects. The relatively weak synergistic effects of rolipram and training may be due to the fact that the dynamics of the signaling cascade leading to phosphorylation of C/EBP, in which increases in cAMP levels enhance PKA activity and, in parallel, ERK is activated, do not appear to be governed by substantial feedback interactions, at least for the protocols used here. Greater synergistic interactions with pharmacological treatments are likely to occur when more complex biochemical elements, such as feedback loops, play a substantial dynamic role (Yin et al., 2014; Zhang et al., 2014). Feedback and cross-talk interactions affecting CREB1 and CREB2 expression do play a role at later times, during the consolidation phase of LTF (Mohamed et al., 2005; Song et al., 2007; Liu et al., 2011a), but they may not contribute to the synergism exhibited by the protocols used here to induce LTF.

\section{Limitations of the model}

Although simulations predicted that the rescue protocol would completely restore LTF impaired by CREB1 knockdown (Fig. $2 B$; taking peak $\mathrm{pC} / \mathrm{EBP}$ as a predictor for the amount of LTF), subsequent experiments showed that the rescue protocol only partially rescued the impaired LTF (Fig. 4). This discrepancy is likely due to the fact that the current model is incomplete. It does not include the intermediate and long-term active phases of PKA, the persistent active phase of ERK, nor the activation of protein kinase $C$ zeta or protein kinase $M$, all of which contribute to the cascade of events underlying LTF (Müller and Carew 1998; Sharma et al., 2003; Cai et al., 2011). For example, CREB1 itself is

B
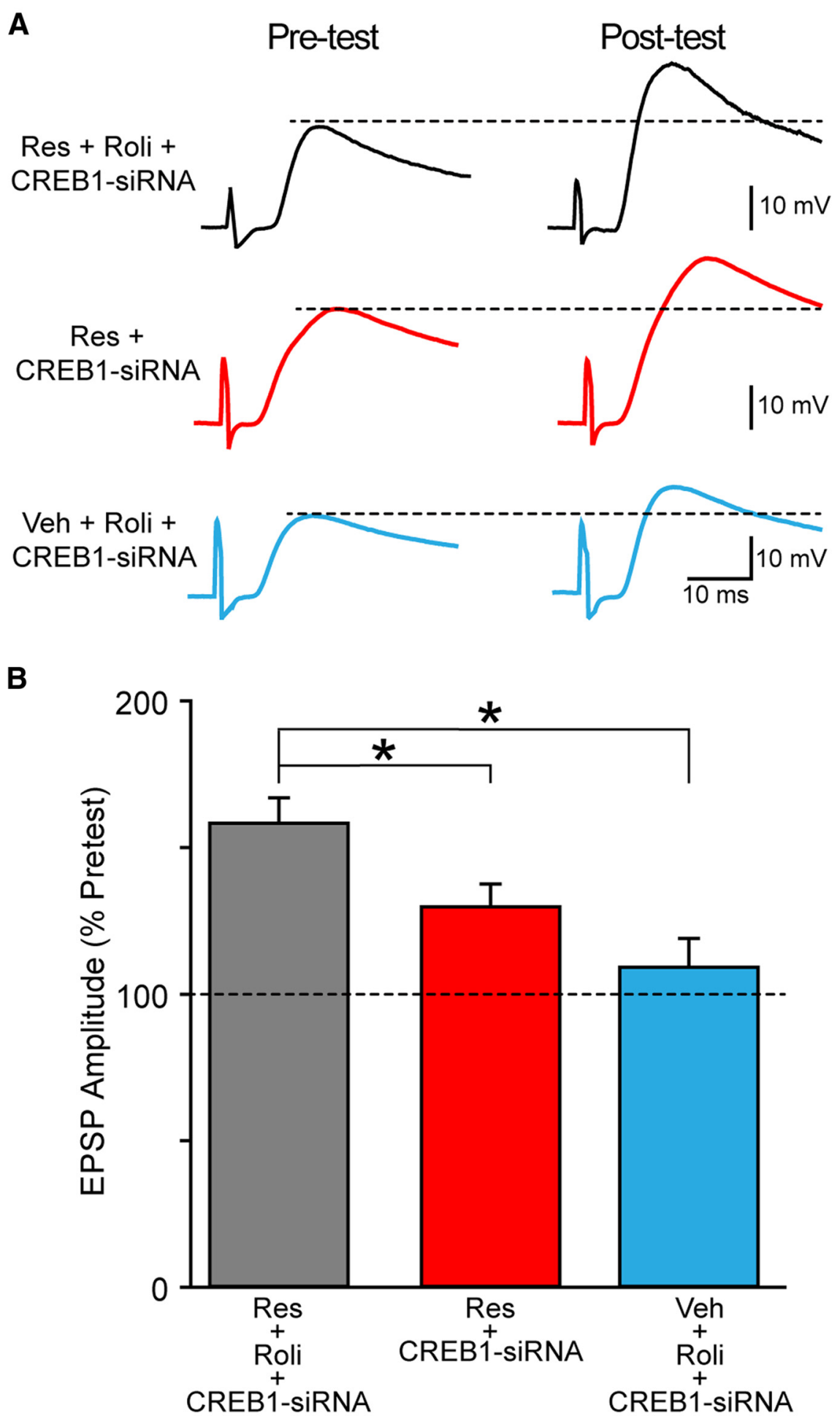

Figure 6. Rolipram enhanced the effectiveness of the rescue protocol in restoring impaired LTF. $A$, Representative traces of EPSPs immediately before (pretest) and $24 \mathrm{~h}$ after (post-test) the rescue protocol. SNs were injected with (REB1 siRNA $72 \mathrm{~h}$ before the pretest. Restoration of LTF by rolipram and/or the rescue protocol was examined in three treatment conditions: (1) the rescue protocol with rolipram (black trace), (2) the rescue protocol without rolipram (red trace), and (3) vehicle treatment with rolipram (blue trace). $\boldsymbol{B}$, Summary data. Error bars represent the mean \pm SEM. A one-way ANOVA indicated no significant differences in the amplitude of pretest EPSPs among these groups $\left(F_{(2,27)}=0.87, p=0.43\right) .{ }^{*} p<0.05$. upregulated during the consolidation phase, and this upregulation contributes to LTF (Liu et al., 2011a). Consequently the empirical knockdown of CREB1 with siRNA would not only reduce basal levels of CREB1, but also its late induction, with this 
later effect not captured by the model. Thus, it is likely that empirically the rescue protocol did not completely compensate for impairment of CREB1 function at these later times. In addition, the dynamics of CREB2 are regulated (Liu et al., 2011b; Rajasethupathy et al. 2012). Variants of the canonical cAMP response element, to which CREB1 and CREB2 bind, are present in the creb1 and creb2 promoters (Mohamed et al., 2005), suggesting that both positive and negative feedback loops play a role in CREB1 and CREB2 dynamics. More precise prediction of rescue protocols may be feasible after the model is extended to include these feedback loops and other processes underlying the induction and consolidation of LTF.

\section{References}

Alarcón JM, Malleret G, Touzani K, Vronskaya S, Ishii S, Kandel ER, Barco A (2004) Chromatin acetylation, memory, and LTP are impaired in CBP $+/-$ mice: a model for the cognitive deficit in Rubinstein-Taybi syndrome and its amelioration. Neuron 42:947-959. CrossRef Medline

Alberini CM, Ghirardi M, Metz R, Kandel ER (1994) C/EBP is an immediate-early gene required for the consolidation of long-term facilitation in Aplysia. Cell 76:1099-1114. CrossRef Medline

Aytan N, Choi JK, Carreras I, Kowall NW, Jenkins BG, Dedeoglu A (2013) Combination therapy in a transgenic model of Alzheimer's disease. Exp Neurol 250:228-238. CrossRef Medline

Barad M, Bourtchouladze R, Winder DG, Golan H, Kandel E (1998) Rolipram, a type IV-specific phosphodiesterase inhibitor, facilitates the establishment of long-lasting long-term potentiation and improves memory. Proc Natl Acad Sci U S A 95:15020-15025. CrossRef Medline

Barrett RM, Malvaez M, Kramer E, Matheos DP, Arrizon A, Cabrera SM, Lynch G, Greene W, Wood MA (2011) Hippocampal focal knockout of CBP affects specific histone modifications, long-term potentiation, and long-term memory. Neuropsychopharmacology 26:1545-1556. CrossRef Medline

Bartsch D, Ghirardi M, Skehel PA, Karl KA, Herder SP, Chen M, Bailey CH, Kandel ER (1995) Aplysia CREB2 represses long-term facilitation: relief of repression converts transient facilitation into long-term functional and structural change. Cell 83:979-992. CrossRef Medline

Bartsch D, Casadio A, Karl KA, Serodio P, Kandel ER (1998) CREB1 encodes a nuclear activator, a repressor, and a cytoplasmic modulator that form a regulatory unit critical for long-term facilitation. Cell 95:211-223. CrossRef Medline

Bourtchouladze R, Lidge R, Catapano R, Stanley J, Gossweiler S, Romashko D, Scott R, Tully T (2003) A mouse model of Rubinstein-Taybi syndrome: defective long-term memory is ameliorated by inhibitors of phosphodiesterase 4. Proc Natl Acad Sci U S A 100:10518-10522. CrossRef Medline

Bourtchuladze R, Frenguelli B, Blendy J, Cioffi D, Schutz G, Silva AJ (1994) Deficient long-term memory in mice with a targeted mutation of the cAMP-responsive element-binding protein. Cell 79:59-68. CrossRef Medline

Cai D, Pearce K, Chen S, Glanzman DL (2011) Protein kinase M maintains long-term sensitization and long-term facilitation in Aplysia. J Neurosci 31:6421-6431. CrossRef Medline

Chain DG, Casadio A, Schacher S, Hegde AN, Valbrun M, Yamamoto N, Goldberg AL, Bartsch D, Kandel ER, Schwartz JH (1999) Mechanisms for generating the autonomous cAMP-dependent protein kinase required for long-term facilitation in Aplysia. Neuron 22:147-156. CrossRef Medline

Dash PK, Hochner B, Kandel ER (1990) Injection of the cAMP-responsive element into the nucleus of Aplysia sensory neurons blocks long-term facilitation. Nature 345:718-721. CrossRef Medline

Gong B, Vitolo OV, Trinchese F, Liu S, Shelanski M, Arancio O (2004) Persistent improvement in synaptic and cognitive functions in an Alzheimer mouse model after rolipram treatment. J Clin Invest 114:1624-1634. CrossRef Medline

Guan Z, Giustetto M, Lomvardas S, Kim JH, Miniaci MC, Schwartz JH, Thanos D, Kandel ER (2002) Integration of long-term memory-related synaptic plasticity involves bidirectional regulation of gene expression and chromatin structure. Cell 111:483-493. CrossRef Medline

Guan Z, Kim JH, Lomvardas S, Holick K, Xu S, Kandel ER, Schwartz JH
(2003) p38 MAP kinase mediates both short-term and long-term synaptic depression in Aplysia. J Neurosci 23:7317-7325. Medline

Josselyn SA, Shi C, Carlezon WA Jr, Neve RL, Nestler EJ, Davis M (2001) Long-term memory is facilitated by cAMP response element-binding protein overexpression in the amygdala. J Neurosci 21:2404-2412. Medline

Kawahara K, Seunobu M, Ohtsuka H, Kuniyasu A, Sugimoto Y, Nakagomi M, Fukasawa H, Shudo K, Nakayama H (2014) Cooperative therapeutic action of retinoic acid receptor and retinoid $\mathrm{X}$ receptor agonists in a mouse model of Alzheimer's disease. J Alzheimers Dis 42:587-605. CrossRef

Korzus E, Rosenfeld MG, Mayford M (2004) CBP histone acetyltransferase activity is a critical component of memory consolidation. Neuron 42:961972. CrossRef Medline

Liu RY, Cleary LJ, Byrne JH (2011a) The requirement for enhanced CREB1 expression in consolidation of long-term synaptic facilitation and longterm excitability in sensory neurons of Aplysia. J Neurosci 31:6871-6879. CrossRef Medline

Liu RY, Shah S, Cleary LJ, Byrne JH (2011b) Serotonin- and traininginduced dynamic regulation of CREB2 in Aplysia. Learn Mem 18:245249. CrossRef Medline

Liu RY, Zhang Y, Baxter DA, Smolen P, Cleary LJ, Byrne JH (2013) Deficit in long-term synaptic plasticity is rescued by a computationally predicted stimulus protocol. J Neurosci 33:6944-6949. CrossRef Medline

Loewe S (1953) The problem of synergism and antagonism of combined drugs. Arzneimittel-Forschung 3:285-290. Medline

MacKenzie SJ, Houslay MD (2000) Action of rolipram on specific PDE4 cAMP phosphodiesterase isoforms and on the phosphorylation of cAMPresponse-element-binding protein $(\mathrm{CREB})$ and $\mathrm{p} 38$ mitogen-activated protein (MAP) kinase in U937 monocytic cells. Biochem J 347:571-578. CrossRef Medline

Martin KC, Michael D, Rose JC, Barad M, Casadio A, Zhu H, Kandel ER (1997) MAP kinase translocates into the nucleus of the presynaptic cell and is required for long-term facilitation in Aplysia. Neuron 18:899-912. CrossRef Medline

Mohamed HA, Yao W, Fioravante D, Smolen PD, Byrne JH (2005) cAMPresponse elements in Aplysia creb1, creb2, and Ap-uch promoters: implications for feedback loops modulating long term memory. J Biol Chem 280:27035-27043. CrossRef Medline

Montarolo PG, Goelet P, Castellucci VF, Morgan J, Kandel ER, Schacher S (1986) A critical period for macromolecular synthesis in long-term heterosynaptic facilitation in Aplysia. Science 234:1249-1254. CrossRef Medline

Müller U, Carew TJ (1998) Serotonin induces temporally and mechanistically distinct phases of persistent PKA activity in Aplysia sensory neurons. Neuron 21:1423-1434. CrossRef Medline

Novick SJ (2013) A simple test for synergy for a small number of combinations. Stat Med 32:5145-5155. CrossRef Medline

Park H, Lee JA, Lee C, Kim MJ, Chang DJ, Kim H, Lee SH, Lee YS, Kaang BK (2005) An Aplysia type 4 phosphodiesterase homolog localizes at the presynaptic terminals of Aplysia neuron and regulates synaptic facilitation. J Neurosci 25:9037-9045. CrossRef Medline

Peters M, Bletsch M, Catapano R, Zhang X, Tully T, Bourtchouladze R (2009) RNA interference in hippocampus demonstrates opposing roles for CREB and PP1 $\alpha$ in contextual and temporal long-term memory. Genes Brain Behav 8:320-329. CrossRef Medline

Philips GT, Kopec AM, Carew TJ (2013) Pattern and predictability in memory formation: from molecular mechanisms to clinical relevance. Neurobiol Learn Mem 105:117-124. CrossRef Medline

Pittenger C, Huang YY, Paletzki RF, Bourtchouladze R, Scanlin H, Vronskaya S, Kandel ER (2002) Reversible inhibition of CREB/ATF transcription factors in region CA1 of the dorsal hippocampus disrupts hippocampusdependent spatial memory. Neuron 34:447-462. CrossRef Medline

Rajasethupathy P, Antonov I, Sheridan R, Frey S, Sander C, Tuschl T, Kandel ER (2012) A role for neuronal piRNAs in the epigenetic control of memory-related synaptic plasticity. Cell 149:693-707. CrossRef Medline

Raymond FL, Tarpey P (2006) The genetics of mental retardation. Hum Mol Genet 15 [Suppl 2]:R110-R116. Medline

Restivo L, Tafi E, Ammassari-Teule M, Marie H (2009) Viral-mediated expression of a constitutively active form of CREB in hippocampal neurons increases memory. Hippocampus 19:228-234. CrossRef Medline 
Roelfsema JH, Peters DJ (2007) Rubinstein-Taybi syndrome: clinical and molecular overview. Expert Rev Mol Med 9:1-16. Medline

Saura CA, Valero J (2011) The role of CREB signaling in Alzheimer's disease and other cognitive disorders. Rev Neurosci 22:153-169. Medline

Sharma SK, Sherff CM, Shobe J, Bagnall MW, Sutton MA, Carew TJ (2003) Differential role of mitogen-activated protein kinase in three distinct phases of memory for sensitization in Aplysia. J Neurosci 23:3899-3907. Medline

Song H, Smolen P, Av-Ron E, Baxter DA, Byrne JH (2007) Dynamics of a minimal model of interlocked positive and negative feedback loops of transcriptional regulation by cAMP-response element binding proteins. Biophys J 92:3407-3424. CrossRef Medline

Stenberg JH, Terevnikov V, Joffe M, Tiihonen J, Tchoukhine E, Burkin M, Joffe G (2011) More evidence on proneurocognitive effects of add-on mirtazapine in schizophrenia. Prog Neuropsychopharmacol Biol Psychiatry 35:1080-1086. CrossRef Medline

Stornetta RL, Zhu JJ (2011) Ras and Rap signaling in synaptic plasticity and mental disorders. Neuroscientist 17:54-78. CrossRef Medline

Titus DJ, Sakurai A, Kang Y, Furones C, Jergova S, Santos R, Sick TJ, Atkins CM (2013) Phosphodiesterase inhibition rescues chronic cognitive deficits induced by traumatic brain injury. J Neurosci 33:5216-5226. CrossRef Medline

Wijetunge LS, Chattarji S, Wyllie DJ, Kind PC (2013) Fragile X syndrome: from targets to treatments. Neuropharmacology 68:83-96. CrossRef Medline
Wood MA, Kaplan MP, Park A, Blanchard EJ, Oliveira AM, Lombardi TL, Abel T (2005) Transgenic mice expressing a truncated form of CREBbinding protein (CBP) exhibit deficits in hippocampal synaptic plasticity and memory storage. Learn Mem 12:111-119. CrossRef Medline

Xiao S, Xue H, Li G, Yuan C, Li X, Chen C, Wu HZ, Mitchell P, Zhang M (2012) Therapeutic effects of cerebrolysin added to risperidone in patients with schizophrenia dominated by negative symptoms. Aust N Z J Psychiatry 46:153-160. CrossRef Medline

Yamamoto N, Hegde AN, Chain DG, Schwartz JH (1999) Activation and degradation of the transcription factor C/EBP during long-term facilitation in Aplysia. J Neurochem 73:2415-2423. Medline

Yin N, Ma W, Pei J, Ouyang Q, Tang C, Lai L (2014) Synergistic and antagonistic drug combinations depend on network topology. PLoS One 9:e93960. CrossRef Medline

Zhang Y, Liu RY, Heberton GA, Smolen P, Baxter DA, Cleary LJ, Byrne JH (2012) Computational design of enhanced learning protocols. Nat Neurosci 15:294-297. Medline

Zhang Y, Smolen P, Baxter DA, Byrne JH (2014) Computational analysis of synergism in small molecular network motifs. PLoS Comput Biol 10: e1003524. CrossRef Medline

Zimmermann GR, Lehar J, Keith CT (2007) Multi-target therapeutics: when the whole is greater than the sum of the parts. Drug Discov Today 12:3442. CrossRef Medline 